\title{
Inhibition of patterned cell shape change and cell invasion by Discs large during D rosophila oogenesis
}

\author{
Scott Goode ${ }^{1}$ and Norbert Perimon ${ }^{2}$ \\ Department of Genetics, ${ }^{2}$ Howard Hughes Medical Institute, Harvard M edical School, Boston, M assachusetts 02115 USA
}

\begin{abstract}
D rosophila D iscs large ( $\mathrm{D} / \mathrm{g}$ ) is a tumor suppressor gene whose loss in epithelial tissues causes disrupted cell polarity and increased cell proliferation. A human Dlg homolog hDlg, has been implicated in tumorigenic processes via its association with the product of the Adenomatous Polyposis Coli (APC) gene. We show for the first time that D rosophila Dlg is required to block cell invasion. Loss of dlg activity during oogenesis causes follicle cells to change shape and invade in a pattem similar to border cells, a small population of cells that break from the post-mitotic follicular epithelium during wild-type oogenesis, yet dlg mutant cells have not adopted a border cell fate. Both functional and morphological evidence indicates that cooperation between germ cell and follicle cell Dlg, probably mediated by DIg PDZ domains, is crucial for regulating cell mixing, suggesting a novel developmental mechanism and mode of action for the DIg family of molecules. These findings suggest that DIg does not simply inhibit individual cell behaviors during oogenesis, but rather acts in a developmental pathway essential for blocking cell proliferation and migration in a spatio-temporally defined manner. A model for Dlg action in blocking cell invasion is presented.
\end{abstract}

[Key Words: Cell invasion; cell proliferation; cell shape; Discs large; membrane-associated guanylate kinases (MAGuKs); PDZ domain]

Received June 12, 1997; revised version accepted August 5, 1997.

Adjoining tissues must regulate their barriers during development and adult life. What factors determine whether cells remain in one place, retaining their associations with their neighbors, or dissociate and move elsewhere? If cells move, what determines where they go, where and when they stop, and whether or not they associate with like or unlike cells? These are important questions in understanding tissue morphogenesis, dynamic physiological processes, and pathologies such as metastasis (Hynes and Lander 1992; Gumbiner 1996).

$M$ aintenance of homotypic adhesion plays a crucial role in ensuring that cells do not mix. Blocking E-cadherin function turns cultured cells from noninvasive to invasive, whereas expressing E-cadherin in cancer cells reverts their tumorigenic phenotype (Takeichi 1993; Birchmeier and Behrens 1994). Physiological invasions and pathological metastasis also require adhesion of invasive cells to foreign matrices and heterologous cells (Mareel et al. 1991). What cellular mechanisms underpin the transfer of adhesion from sites of contact between homotypic cells to sites of contact between heterotypic cells?

An outstanding cell biological and genetic system for analyzing the problem of cell mixing and migration is

${ }^{1}$ Corresponding author.

E-MAIL goode@rascal.med.harvard.edu; FAX (617) 432-7688. the movement of a small cluster of post-mitotic follicuIar cells, called border cells (BCs), between germ cells to the Drosophila oocyte (for review, see M ontell 1994; described in Fig. 1). Several molecules have been identified as crucial players in regulating BC migration. The slow border cells (slbo) gene is specifically expressed in BCs and encodes the Drosophila homolog of a vertebrate basic region-leucine zipper transcription factor, CCAAT enhancer binding protein (C/EBP; M ontell et al. 1992). In animals harboring strong sl bo mutations, BCs fail to initiate migration, whereas weaker slbo mutations cause delayed initiation. Thus, slbo appears to play a role in determining the ability of $\mathrm{BCs}$ to disassociate from neighboring epithelial cells, their ability to attach to germ cells, or both of these processes. The breathless (btl ) gene encodes a FGF receptor tyrosine kinase homolog that appears to be a direct transcriptional target of slbo, and mutations in btl dominantly enhance BC migration defects associated with weak slbo mutations (M urphy et al. 1995). Whereas slbo and btl are crucial for triggering $\mathrm{BC}$ differentiation and movement, little is known about the molecules that are involved in positively or negatively regulating the shape changes, migration, and interaction of BC s with germ cells in a specific pattern. We show that Discs large (DIg), known for regulating cell shape in imaginal epithelia (Woods and Bryant 1991), is likely to be such a molecule. 

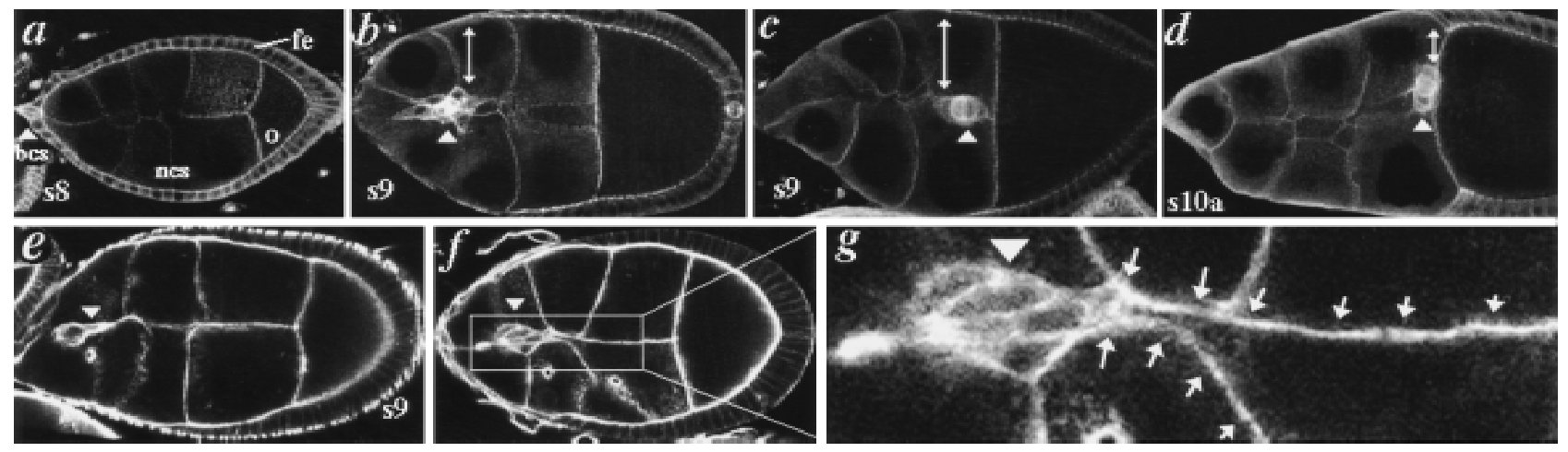

Figure 1. Border cell migration. Arrowheads point to BCs in each panel. (a-d) Expression of Armadillo (Arm) $\beta$-catenin from s8 to s10a. Arm is enriched in BCs before and during their migration through nurse cells (ncs) to the oocyte (o), in concert with the follicular epithelium (fe, double-headed arrows). Migration is complete at s10a (d). (e-g) Wild-type s9 egg chambers stained with phalloidin to reveal cellular cortices. (e) N urse cells in this s9 egg chamber have the commonly observed quadrihedral shape. (f) When BCs have moved between anterior and central nurse cells, a posterior nurse cell, connected to the oocyte, extends a cytoplasmic process that contacts the BCs (boxed area). (g) An enlargement of the nurse cell process (arrows).

DIg belongs to a conserved family of proteins termed membrane associated guanylate kinases (MAGuKs; Woods and Bryant 1991, 1993). These proteins share three amino-terminal PDZ domains (named for the family members PSD-95, DIg, and ZO-1), an SH3 domain, and a carboxy-terminal guanylate kinase (GuK) homology domain (for review, see Anderson 1996; Sheng 1996). DIg molecules may bind to cytoskel etal proteins through the SH3 domain, a known adaptor motif mediating direct association with cytoskeletal and signaling molecules (Mayer and Eck 1995). The GuK-homology domain has not been shown to have kinase activity, and is missing two amino acids thought to be crucial for catalytic activity (Woods and Bryant 1993). In Drosophila, the GuK domain has been shown to play a specific role in prohibiting cell proliferation (Woods et al. 1996).

The three PDZ motifs are perhaps the most intriguing aspect of MAGUK structure. Human DIg binds via the third PDZ motif to APC, a commonly mutated tumor suppressor gene in human colon cancer cells (Matsumine et al. 1996). PDZ motifs have al so been shown to bind to the extreme carboxy-terminal tail of several distinct families of signaling receptors and channels in a sequence-specific fashion (Songyang et al. 1997), and genetic evidence for PDZ involvement in receptor localization at specific subcellular sites has been obtained in Caenorhabditis elegans (Simske et al. 1996). PDZ domains have thus emerged as novel adaptor modules for specific protein-protein binding, important for clustering membrane proteins as well as linking signaling molecules in multiprotein complexes at specialized membrane sites (for review, see Anderson 1996; Sheng 1996).

Drosophila DIg is essential for prohibiting cell growth and for maintaining cell adhesion and cell polarity in both embryonic and adult tissues (Gateff 1978; Perrimon 1988; Woods and Bryant 1989, 1990; Woods et al. 1996). In animals harboring dlg null mutations, aberrant cell polarity is revealed in the aberrant organization of both the actin and microtubule cytoskel etons, the transfor- mation of columnar epithelial cells to a apolar morphology, and the delocalized distribution of cell adhesion molecules (Woods et al. 1996). The role of DIg in maintaining cell polarity appears to be separable from its role in proliferation control, because several mutations that el iminate carboxy-terminal sequences, including the entire GuK domain, cause loss of proliferation control, without affecting cell polarity (Woods et al. 1996).

In this paper we show for the first time that DIg is required to block cell invasion and that DIg activity appears to define a novel devel opmental pathway. Starting very early in oogenesis, loss of dlg acti vity causes follicle cells to overproliferate at the poles of the egg chamber and invade germ tissue. Invading follicle cells change shape and move in a pattern similar to $\mathrm{BC}$, suggesting that the BC migration pathway is established very early in oogenesis. Both functional and morphological evidence indicates that cooperation between germ cell and follicle cell DIg, probably mediated by DIg PDZ domains, is crucial for regulating cell mixing. On the basis of these findings, we suggest a model for DIg action in prohibiting interactions between tissue layers during oogenesis, and for releasing this prohibition in a regulated manner when BCs migrate. We propose that DIg does not simply inhibit individual cell behaviors during oogenesis, but rather acts in a developmental pathway essential for blocking cell proliferation, shape change, and migration in a spatio-temporally defined manner.

\section{Results}

$\mathrm{BC}$ migration

Because our analysis of DIg function during oogenesis focuses on BC migration, we describe and extend previous observations of this process relevant to our studies. T wo postmitotic follicle cell populations mi grate at s9 of oogenesis (Fig. 1; see Fig. 5, below, for stages of oogenesis). Most follicle cells surrounding nurse cells move to 
the oocyte as an epithelial sheet along the outside of the egg chamber, whereas the BCs move through the center of the egg chamber, in concert with the epithelium. Six to seven BCs break from the follicular epithelium and adopt a mesenchymal-like morphology, then migrate to the oocyte, as they contact anterior nurse cells, then posterior nurse cells, before reaching their destination (Fig. 1).

The interaction of BCs with posterior nurse cells is particularly dramatic. N urse cells maintain an invariant quadrihedral-like architecture throughout most stages of oogenesis (Fig. 1e; Fig. 5b, below), but following initiation of BC movement, one nurse cell adjacent to the oocyte extends a cytoplasmic process that contacts the migrating cells (Fig. If,g). N urse cell processes are never observed in s8 egg chambers, preceding BC migration, although BC fate has al ready been established (data not shown; see also Fig. 4, below). Further, nurse cell processes show a clear directionality. They are never observed to extend from anterior nurse cells to BCs that have moved close to the oocyte at the posterior of the egg chamber. These observations suggest that nurse cells adjacent to the oocyte play an active role in guiding the movement of BCs to the oocyte, a suggestion further supported by analysis described below.

Reduction of dlg activity leads to an invasive phenotype starting as early as sl of oogenesis

To anal yze the function of dlg during oogenesis, we took advantage of temperature-sensitive allele combinations (Perrimon 1988). dlg ${ }^{\text {hf321 }} / \mathrm{dlg}^{\mathrm{lv} 55}$ animals are viable and have completely normal egg chambers at $18^{\circ} \mathrm{C}$. When these animals are shifted to $25^{\circ} \mathrm{C}$ for at least $6 \mathrm{hr}$, follicle cells exit the follicular epithelium and intermingle with germ cells at the anterior and posterior poles of the egg chamber (Fig. 2e; data not shown). Cells that remain in the epithelium retain their polarized, epithelial characteristics, whereas cells that have exited the epithelium have an apolar morphology. Follicle cells that have exited the epithelium in anterior regions of the egg chamber invade between nurse cells as they migrate towards the oocyte (Fig. 2, cf. e and f). The phenotype is evident as early as sl of oogenesis, just following the birth of new egg chambers (Fig. 2d).

Several morphological characteristics suggest that dlg invasive cells behave like BCs during wild-type oogenesis. First, BCs always migrate as a cluster of interconnected cells (Fig. 1), and likewise, dlg invasive cells always migrate as streams of interconnected cells (Fig. 2df). Furthermore, like BCs, invasive cells al ways migrate through the center of the egg chamber. They never diverge on their path to move between lateral nurse cell membranes (Figs. 2-4, 6, 7, and 9). That invasive cells are attracted to the oocyte is further supported by simple temperature-shift experiments. Temperature shifts of increasing duration result in increasingly larger streams of follicle cells that move increasingly closer to the oocyte (data not shown). Furthermore, if $\mathrm{dlg}^{\text {hf } 321} / \mathrm{dlg}^{\mathrm{lv} 55}$ animals are shifted to the restrictive temperature for $6 \mathrm{hr}$,

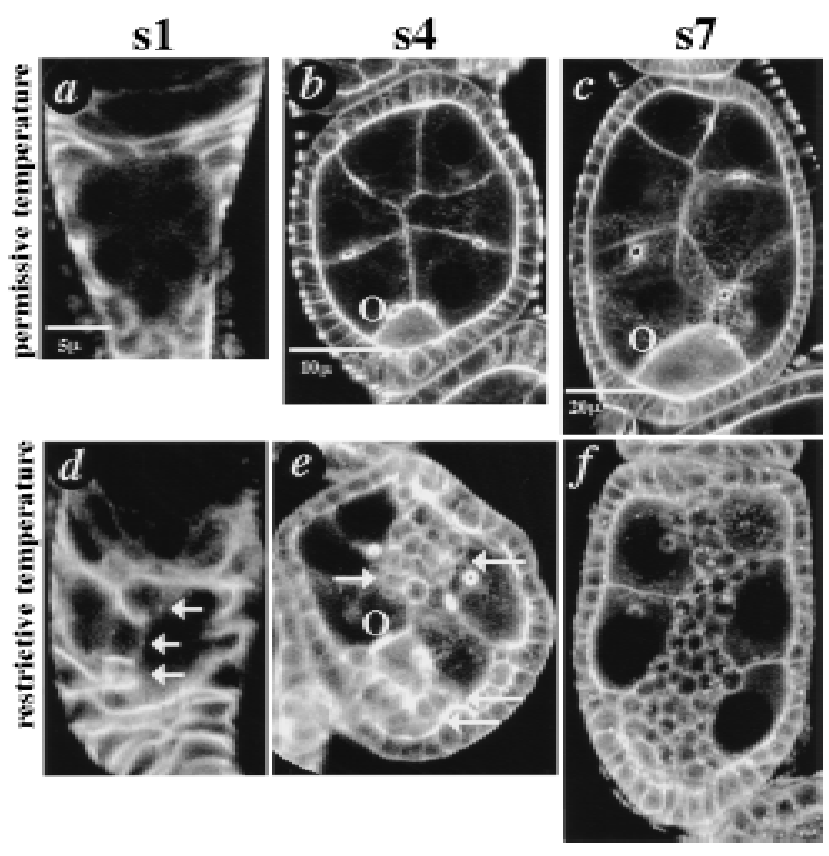

Figure 2. dlg invasive phenotypes. Invasive phenotypes were anal yzed as described in the text. Confocal microscopy was used to capture single optical sections through the center of egg chambers that had been stained with fluorescein-label ed probes. Egg chambers are not shown to scale (see scale bars and Fig. 5;

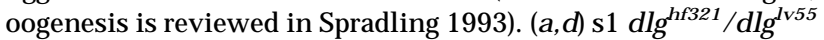
egg chambers were stained with Faslll to reveal follicle cell outlines. (a) A sl egg chamber that devel oped at $18^{\circ} \mathrm{C}$ for several days appears wild type. (d) An egg chamber that developed at $25^{\circ} \mathrm{C}$ for 2 days. Three follicle cells have migrated through the center of the egg chamber along the presumptive BC pathway (arrows). (b,c,e,f) s4 and s7 $\mathrm{dlg}^{\text {hf } 321} / \mathrm{dlg}^{\mathrm{l} v 55}$ egg chambers were stained with phall oidin to reveal cellular cortices. $(b, c) s 4$ and s7 egg chambers that developed at $18^{\circ} \mathrm{C}$ for several days appear wild type. (e) s4 egg chamber that developed at $25^{\circ} \mathrm{C}$ for $18 \mathrm{hr}$. A polar follicle cells have accumulated around the oocyte at the posterior pole of the egg chamber (arrows; O, oocyte), and are invading from the anterior pole (arrows). (f) s7 egg chamber that developed at $25^{\circ} \mathrm{C}$ for $36 \mathrm{hr}$. Follicle cells have invaded germ tissue in a pattern resembling $\mathrm{BC}$ migration.

then placed at the permissive temperature for several hours, a small clump of follicle cells separates from the anterior epithelium; 24 hr later, the small clump of cells is typically found adjacent to the oocyte, and never outside the center of the egg chamber (data not shown).

The same invasi ve phenotype, with varying degrees of severity, is found for many dlg mutant combinations (Table 2, below). The degree of severity directly correlates with that described for imaginal disc tissue (Table 1; Woods et al. 1996). For example, the stage at which the dlg invasive phenotype is first manifested in $\mathrm{dlg}^{\text {hf321 }}$ / dlg $^{1 P 20}$ (or v59 or X1-2) animal s di rectly correlates with the degree of GuK truncation associated with the $\mathrm{dlg}^{1 \mathrm{P} 20}$, $\mathrm{dlg}^{\mathrm{v} 59}$, and dlg ${ }^{\times 1-2}$ mutations (T able 1), with the weakest allele combinations only disrupting the latest stages of oogenesis, and increasingly stronger al lele combinations disrupting increasing earlier stages of oogenesis. A simi- 
Table 1. Characteristics of dlg mutations used in this study

\begin{tabular}{|c|c|c|c|c|}
\hline Mutation & $\begin{array}{l}\text { Invasive } \\
\text { phenotype }\end{array}$ & $\begin{array}{c}\text { Genetic } \\
\text { characteristic }\end{array}$ & $\begin{array}{l}\text { Phenotypic } \\
\text { characteristic }^{c}\end{array}$ & $\begin{array}{c}\text { Molecular } \\
\text { characteristic }^{d}\end{array}$ \\
\hline m52 & yes & $\begin{array}{l}\text { zygotic lethal, behaves like a } \\
\text { null, fails to complement } \\
\text { all dlg mutations }\end{array}$ & loss of PC, CP, and SJ structure & $\begin{array}{l}\text { truncated protein comprised of } \\
\text { PDZ1 and PDZ2, expressed at very } \\
\text { low levels compared to wild type }\end{array}$ \\
\hline hf321 & yes & zygotic lethal, plf $\mathrm{ts}^{\mathrm{g}}$ & N.D. & $\begin{array}{l}\text { 5.5-kb insert at the } 5^{\prime} \text { end of the gene; } \\
\text { effect on Dlg protein not known }\end{array}$ \\
\hline sw & no & viable, plf & loss of PC; CP and SJs normal & $\begin{array}{l}\text { carboxyl-end protein truncation, just } \\
\text { outside of the GKD }\end{array}$ \\
\hline 1P20 & N.D. & zygotic lethal, plf & loss of PC; CP and SJs normal & $\begin{array}{l}\text { eliminates the carboxyl third of the } \\
\text { GKD }\end{array}$ \\
\hline Iv55 & no & zygotic lethal, plf & Ioss of PC; CP and SJs normal & not characterized \\
\hline v59 & no & zygotic lethal, plf & Ioss of PC; CP and SJs normal & $\begin{array}{l}\text { eliminates the carboxyl two-thirds } \\
\text { of the GKD, reduces protein levels } \\
\text { by half }\end{array}$ \\
\hline $\mathrm{X} 12$ & no & zygotic lethal, plf & loss of PC; CP and SJs normal & truncated protein lacking the GKD \\
\hline m35 & no & zygotic lethal, plf & Ioss of PC; CP and SJs normal & $\begin{array}{l}\mathrm{L}_{632}>\mathrm{P} \text { missense mutation in the } \\
\mathrm{SH} 3 \text { domain }\end{array}$ \\
\hline
\end{tabular}

aThe mutations $\mathrm{m} 52, \mathrm{v} 55, \mathrm{v} 59, \mathrm{X} 12$, and $\mathrm{m} 35$ were assayed by the germ cell-follicle cell clone technique described in the text; hf321 was assayed by rearing homozygous animals at $18^{\circ} \mathrm{C}$, the permissive temperature, then shifting them to $25^{\circ} \mathrm{C}$, the restrictive temperature; sw is a female sterile allele that was directly assayed in homozygous females.

bThe genetic characteristics of the mutations used in this study are described in detail in several reports (Perrimon 1988; Woods and Bryant 1989, 1991; Woods et al. 1996).

'Effect of dlg mutations on imaginal and salivary gland epithelial cells (Woods and Bryant 1989, 1991; Woods et al. 1996).

${ }^{\mathrm{d}} \mathrm{M}$ olecular characteristics were established in several publications (Woods and Bryant 1989, 1991; Woods et al. 1996).

e(PC) Proliferation control; (CP) cell polarity; (SJ) septate junction.

${ }^{f}$ (plf) Partial loss of function.

g(ts) Temperature sensitive.

h(GKD) Guanylate kinase domain.

Iar temporally graded pattern of phenotypic expression has been observed for mutations disrupting components of other signaling and adhesion pathways during oogenesis (Goode et al. 1992, 1996a,b). Because a similar invasive phenotype is observed in many dlg mutant combinations, including clones of a null dlg mutation (see below), we conclude that the invasive phenotype is caused by a reduction or loss of dlg activity.

Invasive follicle cells have not adopted a $\mathrm{BC}$ fate

Because dl $\mathrm{g}^{\text {hf321 }} / \mathrm{dlg}^{\mathrm{lv} 55}$ foll icl e cells behave like BCs, we determined whether they have adopted a BC fate. The sl bo gene (M ontell et al. 1992) is specifical ly expressed in $\mathrm{BC} s$ at $\mathrm{s8}$, before they break from the epithelium and initiate migration to the oocyte, and sl bo continues to be expressed in $\mathrm{BC} s$ during their migration to the oocyte at s9 (Fig. 3a-C). We analyzed the expression of sl bo in dlg mutant egg chambers. slbo is expressed in the wild-type pattern in s8 and s9 $\mathrm{dlg}^{\text {hf }} 321 / \mathrm{dlg}^{\mathrm{lv55}}$ mutant egg chambers and is not expressed in invasive follicle cells (Fig. 3d,e). sl bo expressi on never initiates before s8 in dl g mutant animal s (not shown). We al so anal yzed expression of FasllI (Patel et al. 1987), which is specifically expressed in two polar cells at the anterior of the egg chamber starting during mid oogenesis. The polar cells are included among the cells that will go on to migrate as border cells (not shown). We did not find an increase in the number of Faslll-positive cells in $\mathrm{dlg}^{\mathrm{hf} 321} / \mathrm{dlg}^{\mathrm{lv55}} \mathrm{mu}$ - tant egg chambers, or in egg chambers harboring follicle cell clones of the null dl $\mathrm{g}^{\mathrm{m} 52}$ mutation, further suggesting that overaccumulating follicle cells have not acquired a border cell fate (data not shown). Finally, we note that whereas dlg-invasive cells share an apolar morphology with border cells, other aspects of their morphology, such as absence of lamellipodia-like structures (Figs. 1, 2), are not shared with border cells. On the basis of these observations, we conclude that dlg invasive follicle cells have not adopted a BC fate, yet, as described below, these cells undergo and participate in several morphogenetic transitions characteristic of BC invasion.

The oocyte and nurse cells attached to the oocyte extend processes that contact invasive follicle cells

Although $\mathrm{dlg}^{\text {hf }} 321 / \mathrm{dlg}^{\mathrm{lv} 55}$ follicle cells have not adopted a BC fate, they resemble BCs in their apolar morphology and migration pattern. We sought to determine if dlg follicle cells also behave like BC s in their pattern of interaction with nurse cells attached to the oocyte (Fig. le-g). To perform these experiments, $\mathrm{dlg}^{\mathrm{hf} 321} / \mathrm{dlg}^{\mathrm{lv} \text { 55 }}$ animals were shifted to the restrictive temperature for 6-12 hr, so that invasive follicle cells would initiate migration just past the first nurse cells (Fig. 4a), the point at which BCs come in contact with a process extended by an oocyte-associated nurse cell (Fig. le-g). In dlght321/ $\mathrm{dlg}^{\mathrm{lv55}}$ mutant egg chambers, nurse cells extend processes to meet invasive follicle cells. This is clear by s6, 
Figure 3. Invasive follicle cells are not border cells. Egg chambers harbor a single copy of a lacZ enhancer trap gene inserted in the sl bo gene (M ontell et al. 1992). sl bo expression is revealed by $\mathrm{X}$-gal staining (blue). (a-c) Expression of slbo in wild-type s8-s10 egg chambers. slbo expression initiates at $\mathbf{s} 8$ in a small cluster of presumptive BCs (arrowheads), and is maintained in $\mathrm{BCs}$ as they migrate to the oocyte. At s10, slbo is also expressed in follicle cells in the anterior follicular epithelium that

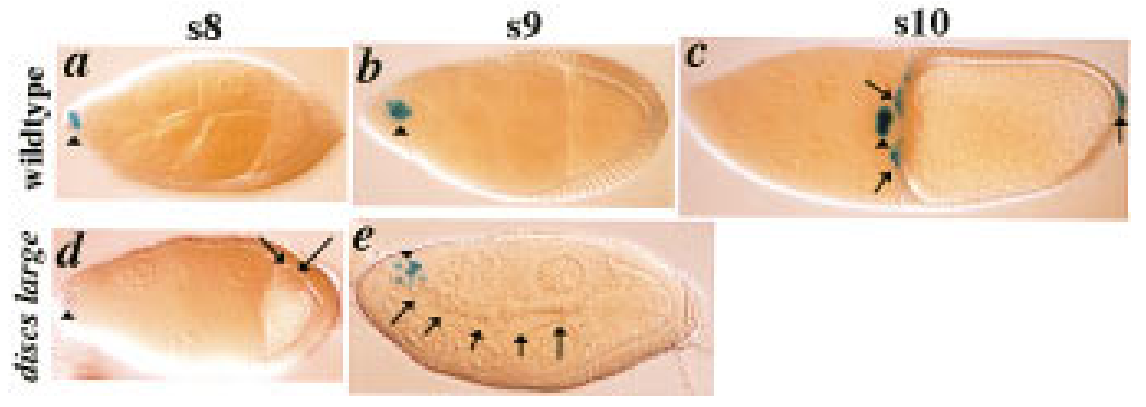
move between the oocyte and nurse cells (arrows), and in a cluster of follicle cells at the posterior of the egg chamber (arrow). (d-e) Expression of slbo in $\mathrm{dlgh}^{\mathrm{hf} 321} / \mathrm{dlg}^{\mathrm{Iv} 55}$ egg chambers. (d) s8 egg chamber that was shifted to the restrictive temperature for $8 \mathrm{hr}$ in which slbo expression has initiated (arrowhead). A small cluster of follicle cells that do not express sl bo have segregated from the epithelium surrounding the ooctye at the posterior of the egg chamber (arrows). (e) A s9 egg chamber that was shifted to the restrictive temperature for $36 \mathrm{hr}$ in which slbo is expressed in border cells as they initiate migration but is not expressed in the stream of invasive cells that have moved to the oocyte (arrows).

when the egg chamber has acquired an elongated shape (Fig. 5a), but as for the invasive phenotype, these interactions probably start as early as s1 (see al so Fig. 2e). We confirmed these interactions for other allele combinations. For example, the interactions are al so observed in $\mathrm{dlg}^{\text {hf321 }} / \mathrm{dlg}^{\text {IP20 }}$ animals, in which cells do not invade before s7 (Fig. 4b; Table 2).

Weal so found that invasi ve follicle cells show unusual interactions with the oocyte. Once invasive cells have migrated to the most posterior nurse cells, the oocyte el ongates to contact the cells (Fig. 4c,d; see al so Fig. 8d). Although the oocyte does not extend during BC migration in wild-type animals, this result documents the selective adhesive forces acting between the oocyte and $\mathrm{BCs}$, and suggests that dlg plays a role in maintaining oocyte architecture during wild-type oogenesis.

\section{DIg expression}

To hel $p$ establish the basis for the invasive behavior of follicle cells in dlg mutant egg chambers, we analyzed the spatio-temporal and subcellular distribution of DIg proteins throughout oogenesis. Western analysis reveals three polypeptide species of about 91, 100, and 102 kD (Fig. 5a), approximately the same mass described for DIg proteins in imaginal discs and larval muscles (Lahey et al. 1994; Woods et al. 1996). DIg is expressed in both germ and follicle cell ti ssues from the time that the germ cell cyst becomes surrounded by follicle cells in the germarium (Fig. 5b). DIg appears to be expressed at equivalent levels in both tissues throughout the growth phases, as the germ cell cyst expands in size and the number of follicular epithelial cells increases $>10$-fold. Following cessation of follicle cell proliferation, levels of DIg protein appear to dramatically decrease in germ cells, corresponding to the time just preceding and including $\mathrm{BC}$ migration to the oocyte (Fig. 5b).

At the cellular level, DIg is local ized to sites of contact between follicle cells and to sites of contact between germ cells, but appears to be excluded at sites of contact between germ cells and follicle cells (Fig. 5c,d). Li kewise, before and during BC migration, DIg is expressed at sites

Figure 4. Posterior germ cells extend processes that contact invasive follicle cells. (a) s6 $\mathrm{dlg}^{\text {hf } 321} / \mathrm{dlg}^{\mathrm{Iv} 55}$ egg chamber shifted to the restrictive temperature for $6 \mathrm{hr}$. N urse cells adjacent to the oocyte extend cytoplasmic processes that contact newly invading follicle cells (outlined). (b) s7 dlghf321/ $\mathrm{dlg}^{1 \mathrm{P} 20}$ egg chamber. A nurse cell attached to the oocyte (via the ring canal, arrow) extends a cytoplasmic process to the anterior pole of the egg chamber (arrowheads), contacting newly invading follicle cells. N ote the resemblance of this process to that extended at 59 during $B C$ migration in wildtype egg chambers (Fig. 1). (c) s8 $\mathrm{dlg}^{\text {hf } 321} /$ dlg $^{\mathrm{lv} 55}$ egg chamber in which invading fol-

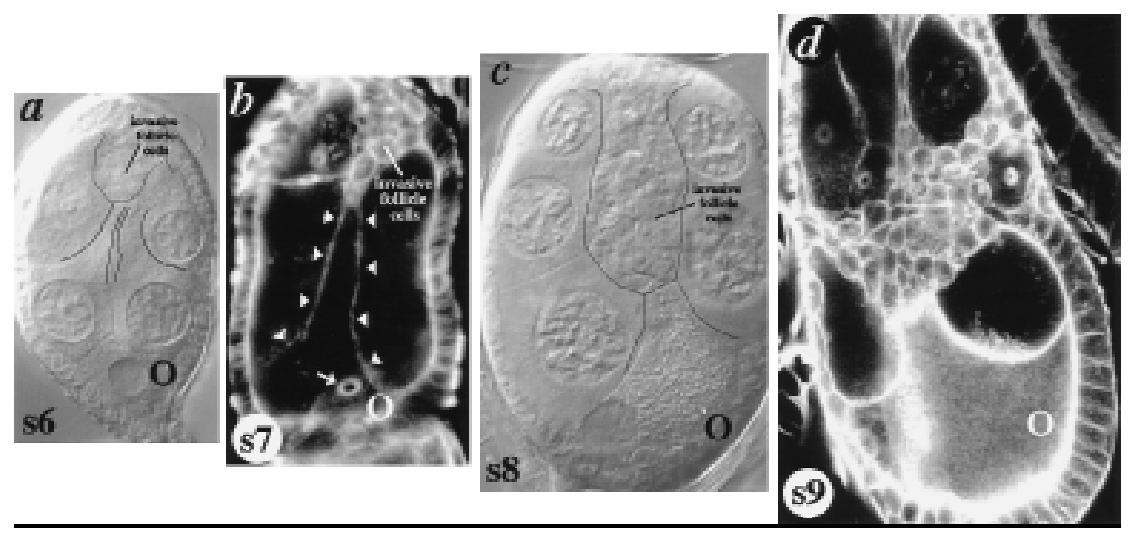

licle cells have migrated over half the distance to the oocyte. The oocyte extends to meet invading cells. (d) Phalloidin-stained s9 egg chamber harboring a presumptive simultaneous germ cell and follicle cell clone of the genetic null dlgm52 mutation (see Fig. 5; $M$ aterials and $M$ ethods). This egg chamber documents a rare instance in which follicle cells invade from lateral portions of the egg chamber, as well as from the anterior pole. The dramatic extension of the oocyte towards the invading cells shows the invading cells attraction to the posterior egg cell. 


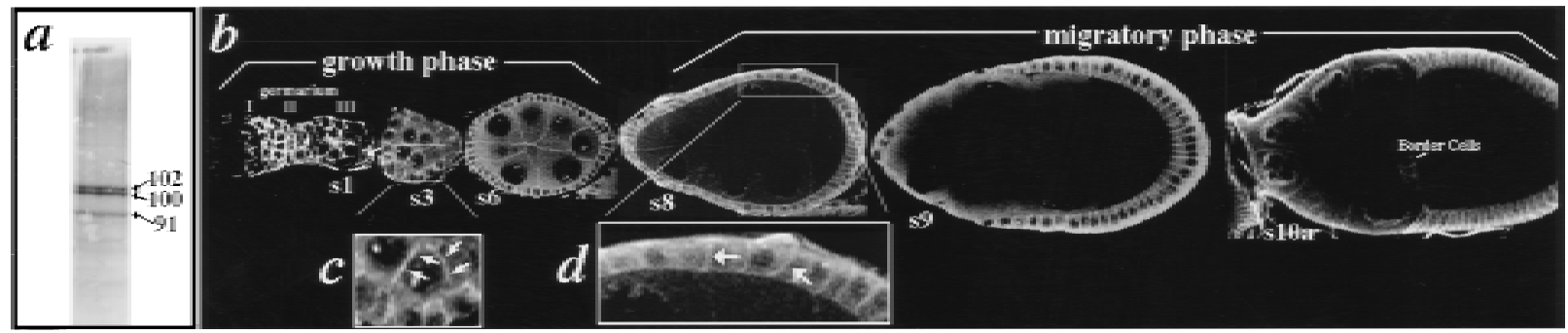

Figure 5. Expression of DIg during oogenesis. (a) Western blot of ovarian proteins probed with DIg sera. Three polypeptides of 91, 100, and 102-kD are detected (arrowheads). (b) Ovarian tissue probed with DIg sera and visual ized by confocal microscopy. DIg is expressed in germ cells and follicle cells starting in the germarium [where the germ cell cyst becomes ensheathed by follicle cells in region II; oogenesis is reviewed in Spradling (1993); cell types are labeled in Fig. 1]. DIg protein expression continues in both cell types throughout the growth stages of oogenesis, as the germ cell cyst expands and the follicular epithelium increases from 40 cells at s1 to 650 cells at s6. DIg staining is detected in both the cytoplasm and membrane. Staining is enriched in membranes at sites of contact between germ cells (c, arrows), and sites of contact between follicle cells (d, arrows). Following cessation of follicle cell division at s6, DIg protein levels dramatically decrease in germ cells during s8-s10 (b), a migratory phase when follicle cell populations move to the oocyte (see also Fig. 1).

of contact between border cells, but not at points of contact between border cells and germ cells (data not shown). Considerable focus has been placed on the localization and function of DIg in epithelial septate junctions (Woods and Bryant 1991, 1993), but our analysis does not address the function of DIg in these structures, because septate junctions are not found in the germ cell cyst or follicular epithelium throughout the stages preceding and including BC migration (M ahowald 1972).

DIg is required in both germ cells and follicle cells

Because DIg is expressed in both germ cells and follicle cells, we sought to determine the respective function of germ cell DIg and follicle cell DIg in the genesis of the invasi ve phenotype. To determine where DIg is required, germ cell and fol licle cell clones were generated with the genetic null mutation dlg ${ }^{m 52}$ (Fig. 6b). Eliminating dlg function in germ cells alone did not cause any defects, except for occasional misshapen germ cells (Fig. 6c, not shown). Eliminating dlg function in follicle cells alone led to overaccumulation of follicle cells at the anterior and posterior poles of the egg chamber (as in dlghf321/ $^{\text {his }}$ $\mathrm{dlg}^{\mathrm{Iv} 55}$ egg chambers, see Fig. 2e), but these cells invade germ tissue only rarely (Fig. 6d; invasion occurs in $\sim 1 / 50$ egg chambers, $n=425$, Fig. 6f). When dlg function is eliminated in both germ cells and follicle cells, follicle cells always invade along the BC pathway (Fig. 6e). We conclude that DIg acts in both germ cells and follicle cells to block cellular invasion.

DIg appears to be required for prohibiting cell proliferation at the poles of egg chambers

We considered two possibilities for the overaccumulation of follicle cells at the poles of dlg mutant egg chambers (Fig. 2e; see below). One possibility is that follicle cells have redistributed from lateral regions of the egg chamber to the egg chamber poles. This does not seem likely because even after $36 \mathrm{hr}$ at the restrictive temperature, and the massive invasion of follicle cells, lateral regions of the follicular epithelium do not appear to differ in cell density from wild-type epithelia (Fig. 2, c and f). A second possibility is that follicle cell overaccumulation results from follicle cell overproliferation.

To obtain evidence for overproliferation of follicle cells, we compared the degree to which follicle cells at the poles of wild-type and mutant egg chambers incor-

Table 2. Phenotypic characteristics of heteroallelic dlg combinations

\begin{tabular}{|c|c|c|}
\hline Strength & Follicle cells invade ${ }^{a}$ & $\begin{array}{l}\text { Follicle cells do not } \\
\text { invade }\end{array}$ \\
\hline weak $^{\mathrm{b}}$ & \multicolumn{2}{|c|}{ sw/m35, sw/hf321 } \\
\hline intermediate $^{c}$ & $\begin{array}{r}\text { 1P20/hf321, } \\
\text { v59/hf321 }\end{array}$ & $\begin{array}{l}\text { 1P20/m35, } \\
\text { Iv55/m35,v59/m35 }\end{array}$ \\
\hline strong $^{d}$ & $\begin{array}{l}\text { X12/hf321, } \\
\text { Iv55/hf321 }\end{array}$ & $\begin{array}{l}\text { sw/sw, } 1 \mathrm{P} 20 / \mathrm{sw} \\
\text { v59/sw, Iv55/sw, } \\
\times 12 / \mathrm{sw}, \mathrm{m} 52 / \mathrm{sw}\end{array}$ \\
\hline lethal $^{e}$ & \multicolumn{2}{|c|}{$\begin{array}{l}\mathrm{hf} 321 / \mathrm{m} 52,1 \mathrm{P} 20 / \mathrm{m} 52, \mathrm{Iv} 55 / \mathrm{m} 52, \mathrm{v} 59 / \mathrm{m} 52 \\
\text { X12/m52, } \mathrm{m} 35 / \mathrm{m} 52, \mathrm{Iv} 55 / \mathrm{v} 59,1 \mathrm{P} 20 / \mathrm{v} 59 \\
\text { X12/v59, 1P20/lv55, X12/lv55, 1P20/X12, } \\
\text { m35/X12, hf321/m35 }\end{array}$} \\
\hline
\end{tabular}

The phenotypes associated with the homozygous mutants is given in Table 1.

alnvasive vs. noninvasive phenotypes are described and pictured in the text.

${ }^{\mathrm{b}} \mathrm{BC}$ s leave prematurely at $\mathrm{s8}$, or move prematurely at s9. Since $\mathrm{BC} s$ are determined at $\mathrm{s} 8$ and migrate between germ cells at $\mathrm{s} 9$, the designation of "Follicle cells invade" or "Follicle cells do not invade" is not meaningful.

'Follicle cells accumulate at the poles and/or invade typically starting no earlier than s5.

${ }^{d}$ Follicle cells accumulate at the poles and/or invade starting at least as early as s3, and for most allele combinations, the earliest time that a mutant phenotype was observed is s1.

ephenotypic analysis not possible because the mutant combinations are lethal at both $18^{\circ} \mathrm{C}$ and $25^{\circ} \mathrm{C}$. 

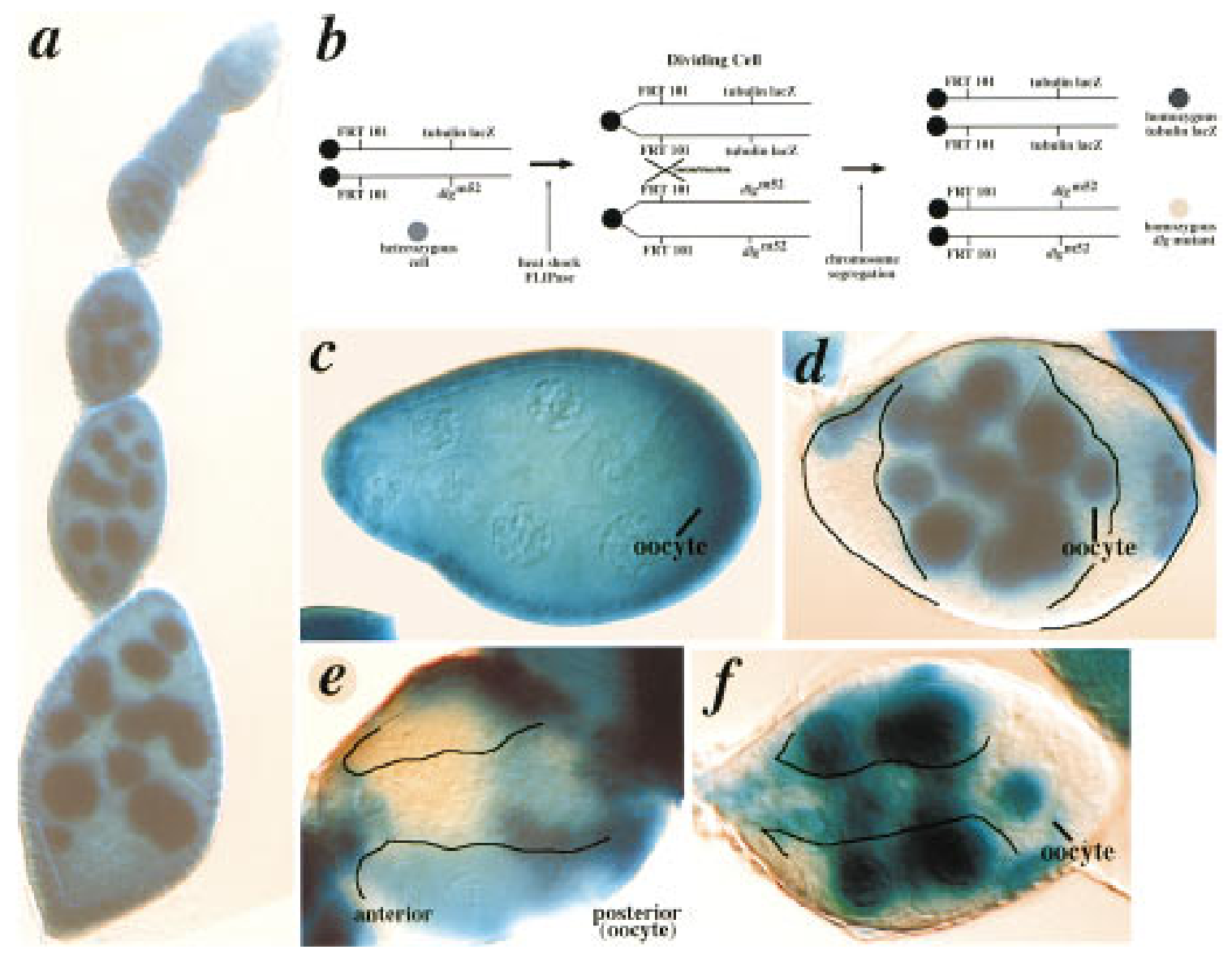

Figure 6. Clonal analysis. Except in rare instances, cell invasion depends on loss of dlg in both germ and follicle cells. (a) X-gal stained ovariole from an FRT ${ }^{101}$, TUBlacZ/ FRT ${ }^{101}$, dl $\mathrm{g}^{\mathrm{m} 52}$; hsFLPase animal. The tubulin promoter drives lacZ expression (TU BlacZ) in both germ and follicle cell nuclei (see $M$ aterials and M ethods). (b) Scheme for generating germ and follicle cell clones (see $M$ aterials and M ethods). Following sister chromatid exchange, homozygous mutant cells do not stain because they no longer harbor the TUBlacZ gene. (c) dl g ${ }^{m 52}$ germ-line clone. Egg chambers with homozygous $\mathrm{dlg}^{\mathrm{m} 52}$ germ cells and heterozygous follicle cells usually appear wild type. (d) dlg ${ }^{\mathrm{m} 52}$ follicle cell clone. Egg chambers with homozygous dl $\mathrm{g}^{\mathrm{m} 52}$ follicle cells and heterozygous germ cells have multiple layers of follicle cells at the anterior and posterior poles (outlined). (e) Egg chamber with homozygous mutant dlgm52 germ cells and follicle cells. Follicle cells invade germ tissue (outlined). (f) dl $\mathrm{g}^{\mathrm{m} 52}$ follicle cell clone. Although germ cells harbor a wild-type copy of dlg, follicle cells invade (outlined). These egg chambers are observed at $\sim 2 \%$ frequency $(n>200)$.

porate BrdU, a marker for newly dividing cells, as a function of distance along the anterior-posterior axis. Wildtype follicle cells incorporate BrdU at the same frequency independent of anterior-posterior position (Fig. $7 \mathrm{a}, \mathrm{c})$. In contrast, the relative frequency with which $\mathrm{dlg}^{\text {hf } 321} / \mathrm{dlg}^{\mathrm{lv55}}$ follicle cells incorporate BrdU is higher at the anterior and posterior poles of the egg chamber (Fig. 7b,c). The difference between mutant and wild type was even more pronounced if the degree of incorporation of BrdU in folliclecells thought to be most susceptible to loss of dlg activity, those residing strictly within the plane of invasive cells, was anal yzed (Fig. 7c).

To further substantiate that follicle cells overproliferate in dlg egg chambers, the total number of follicle cells in $\mathrm{dlg}^{\mathrm{sw}}$ versus wild-type s5-s6 egg chambers was com-

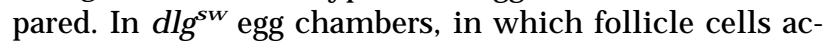
cumulate at the pol es of the egg chamber without invading (Fig. 7d), there are on average 150 more follicle cells per egg chamber compared with wild type (Fig. 7e). Fur- ther, these follicle cells appear to overaccumulate at the anterior and posterior poles of the egg chambers, where we observed increased BrdU incorporation in dlghf321/ dlg $g^{\mathrm{lv5}}$ egg chambers (Fig. 7f). Combined, these findings strongly indicate that BrdU incorporation at the poles of $\mathrm{dlg}^{\text {hf321 }} / \mathrm{dlg}^{\mathrm{lv55}}$ egg chambers results from loss of prol iferation control, and not from a redistribution of follicle cells.

dlg mutations that map to the $\mathrm{SH} 3$ and GuK domains do not confer premature cell mixing

dlg mutations that behave as genetic nulls, such as dlg $^{\mathrm{m} 52}$ (described above), cause loss of proliferation control, cell polarity, and adhesion in imaginal epithelia (Woods et al. 1996; Table 1), and an invasive phenotype when removed from germ cells and follicle cells during oogenesis (Fig. 6). In contrast, dlg mutations that specifically disrupt the $\mathrm{SH} 3$ and GuK domains cause loss of 

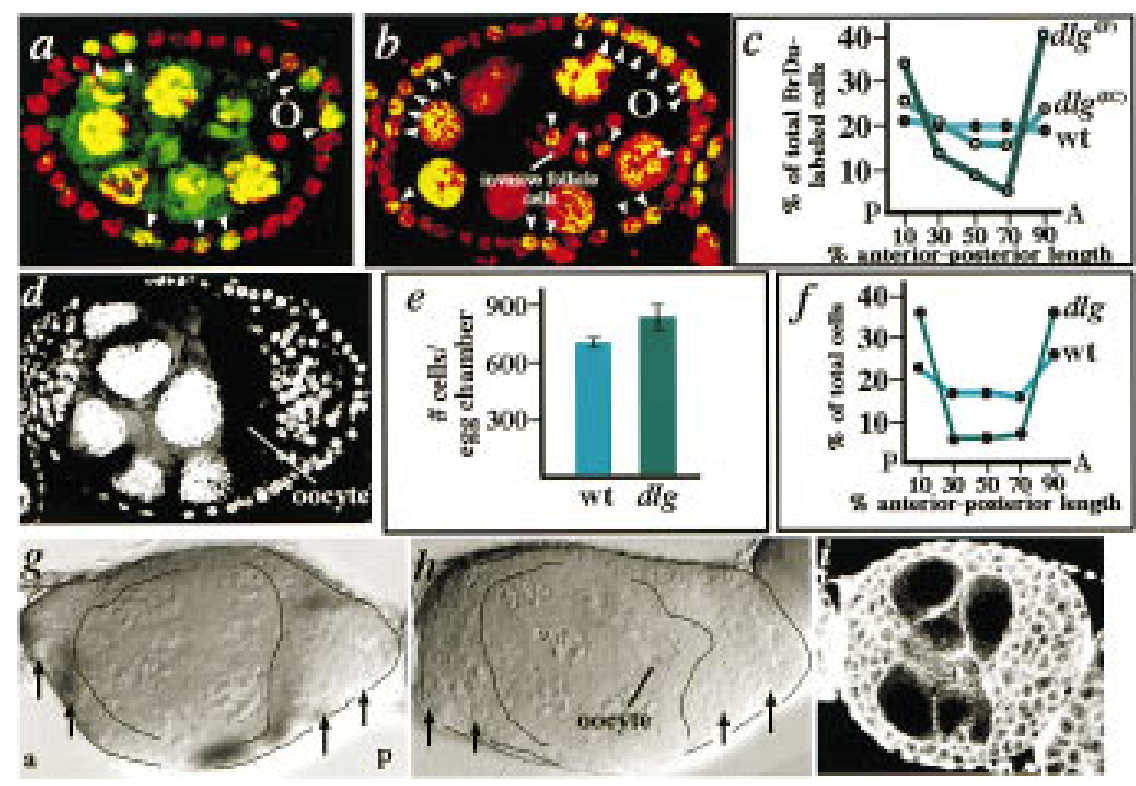

Figure 7. Separation of DIg invasion and proliferation phenotypes. (a-c) Follicle cells at the anterior and posterior poles of $\mathrm{dlg}^{\text {hf } 321} / \mathrm{dl} \mathrm{g}^{\mathrm{lv55}}$ mutant egg chambers preferentially incorporate BrdU. BrdU is a nucleotide anal og that is incorporated into nuclei that are in S-phase, or have entered S-phase during the experimental period. $\mathrm{N}$ ote the intense accumulation of BrdU in nurse cell nuclei (yellow), which are polyploid. (a) s6 wild-type egg chamber. All follicle cells have been labeled with propidium iodide (red nuclei), whereas follicle cells that have incorporated BrdU during the $12 \mathrm{hr}$ experimental period have yellow nuclei (arrow heads; see Materials and M ethods). (b) s6 dl g ${ }^{\text {hf321 }} / \mathrm{dl} \mathrm{g}^{\mathrm{lv55}}$ egg chamber with invading follicle cells migrating to the oocyte. Follicle cells at the poles of the mutant egg chambers appear to have incorporated BrdU to a greater extent than wild type. (c) Graph comparing the average distribution of BrdU-labeled follicle cells at the anterior and posterior poles of wild-type and mutant egg chambers. BrdU-label ed follicle cells are found at about equal frequency al ong the anterior-posterior axis of wild-type egg chambers, but are found at greater frequency at the anterior and posterior poles of

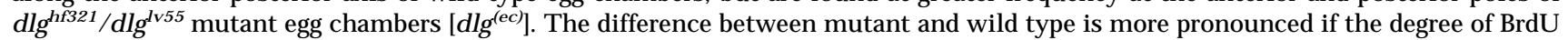
incorporation in follicle cells that reside strictly within the plane that includes invasive cells is analyzed [dl $\mathrm{g}^{(\mathrm{IP})}$ ]. (d-i) Characterization of dlg genotypes and phenotypes in which follicle cells overaccumulate, but do not invade. (d) A dlg ${ }^{\text {sw }}$ egg chamber. Follicle cells accumulate at the poles of the egg chamber, but do not invade. (e) Graph comparing the total number of follicle cells in dlg ${ }^{\text {sw }} /+($ wild type) vs. $\mathrm{dlg}^{\mathrm{sw}}$ egg chambers ( $\mathrm{n}=4$ egg chambers for each genotype). There are $\sim 150$ more follicle cells in mutant egg chambers on average. (f) Graph comparing the distribution of follicle cells in the center of s7 dl g $\mathrm{gw}^{\mathrm{sw}} /+\mathrm{vs}$. the center of $\mathrm{s} 7 \mathrm{dlg} \mathrm{g}^{\mathrm{sw}} / \mathrm{dl} \mathrm{g}^{\mathrm{sw}}$ egg chambers (numbers obtained from confocal sections like those shown in a and d; $n=6$ egg chambers for each genotype). M any more follicle cells are found at the poles of $\mathrm{s} 7 \mathrm{dl} \mathrm{g}^{\mathrm{sw}}$ egg chambers compared with $\mathrm{dl} \mathrm{g}^{\mathrm{sw}} /+$ egg chambers. N ote that supernumerary follicle cells are found in the same position wherever increased BrdU incorporation is found in $\mathrm{dlg}^{\text {hf } 321} / \mathrm{dlg}^{\mathrm{lv55}}$ egg chambers (c). (g,h) Egg chambers harboring simultaneous germ cell and follicle cells clones of $\mathrm{dlg}^{\mathrm{X} 1-2}$ and $\mathrm{dl}^{\mathrm{m} 35}$, respectively (produced as in Fig. 6). Follicle cells accumulate at the poles of egg chambers (outlined, arrows), but do not invade germ tissue. (i) $\mathrm{dlg}^{\mathrm{5} 59} / \mathrm{dl} \mathrm{g}^{\mathrm{m} 35}$ egg chamber stained with phalloidin to reveal actin cortices. Follicle cells overaccumulate at the poles of egg chambers, but do not invade.

proliferation control, but have no effect on cell polarity (Table 1; Woods et al. 1996). To assay the requirement of the $\mathrm{SH} 3$ and GuK domains in blocking cell invasion, we analyzed the phenotype of SH3 and GuK mutations by employing the germ cell-follicle cell clone technique described (Fig. 6). In egg chambers in which both germ cells and follicle cells are mutant for $\mathrm{dlg}^{\mathrm{lv} v 5}$ or $\mathrm{dl}^{\mathrm{g}} \mathrm{I}^{\mathrm{1-2}}$ mutations, which partially and almost completely el iminate the GuK domain, respectively, or for the dlgm30 mutation, which disrupts the $\mathrm{SH} 3$ domain, follicle cells accumulate at the poles of the egg chamber, but do not invade between germ cells (Fig. 7g,h). Further, in animals heterozygous for dl $\mathrm{g}^{\mathrm{IP} 20}$ or Iv55 (that disrupt GuK sequences), and $\mathrm{dl}^{\mathrm{m}} \mathrm{m}^{52}$ (that disrupts the SH 3 domain), follicle cells accumulate at the poles of egg chambers without invading (Table 2; Fig. 7i). These results indicate that DIg molecules lacking the activity of the GuK domain, and probably the activity of the SH3 domain, retain the DIg activity that prohibits follicle cell invasion.

\section{DIg prohibits premature $\mathrm{BC}$ migration}

Our analysis indicated that DIg is required for inhibiting several interactions and activities in growing egg cham- bers that appear to be fundamental to $\mathrm{BC}$ migration. Does DIg play a similar role during the process of $B C$ migration? To analyze the role of dl g during post-mitotic stages, we used extremely weak mutant combinations, such as dl g $g^{\text {hf321 }} / \mathrm{dl} \mathrm{g}^{\text {m35 }}$ and dl $\mathrm{g}^{\text {hf321 }} / \mathrm{dll}^{\text {sw }}$, in which no detectable defect on egg chamber development is found until s8-s9, when BCs are determined and mi grate ( $T$ able 1). At s8, the only observable defect is the occasional premature breaking and movement of BCs from the anterior fol licular epithel ium (Fig. 8a,b). We do not think it is likely that this phenotype results from delayed migration of the follicular epithel ium towards the oocyte, be cause we do not observe defective epithelium migration in stronger dlg mutant combinations. At $s 9$, in addition to the premature arrival of $\mathrm{BCs}$ to the oocyte, we sometimes observe cytoplasmic extensions between the oocyte and BCs, suggesting excessive interaction between these cells (Fig. 8c,d). These extensions are not found during $\mathrm{BC}$ migration in wild-type egg chambers. These results indicate at least two similarities for the role of DIg during BC migration and the earlier stages of oogenesis. First, the premature breaking of BCs suggests a functional connection to the early invasive phenotype, and second, the pulling of the oocyte by the BCs is remi- 


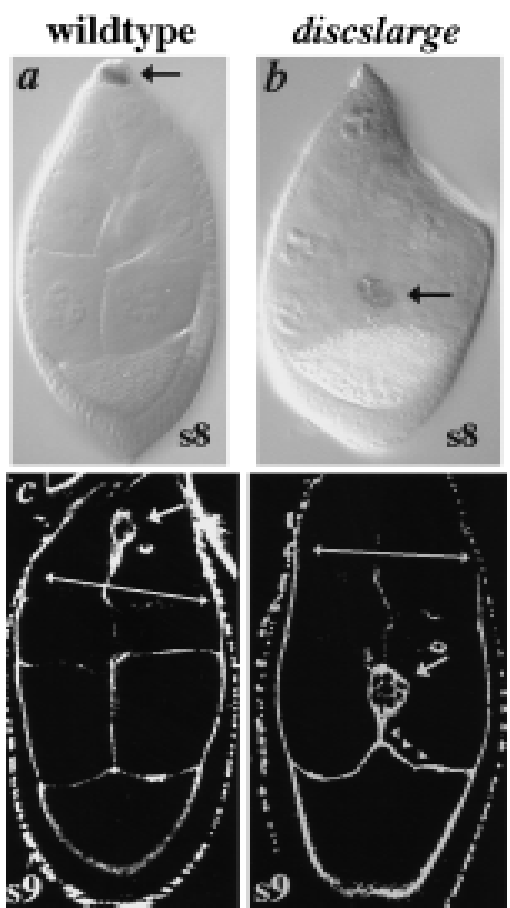

Figure 8. Weak dlg mutations cause BCs to migrate in advance of the follicular epithelium. (a-b) Egg chambers harbor a single copy of a lacZ enhancer trap gene inserted in the slbo gene (M ontell et al. 1992). slbo expression is revealed by X-gal staining of egg chambers that have developed at $25^{\circ} \mathrm{C}$. (a) Typical s9 wild-type egg chamber in which BCs migrate in concert with the follicular epithelium (arrows). (b) Typical s9 $\mathrm{dlg}^{\mathrm{sw}} / \mathrm{dlg}^{\text {hf3321 }}$ egg chamber in which BCs have migrated in advance of the follicular epithelium. This is the only defect observed in the ovaries of $\mathrm{dlg}^{\mathrm{sw}} / \mathrm{dlg}^{\text {hf } 321}$ animals. (c-d) Egg chambers stained with fluorescein-phalloidin and imaged by scanning laser confocal microscopy to reveal cortices of germ and follicle cells. (c) Typical s9 wild-type egg chamber in which BCs migrate in concert with the follicular epithelium (arrows). (d) Typical s9 $\mathrm{dl} \mathrm{g}^{\text {sw }} /$ $\mathrm{dlg}^{\text {hf } 321}$ egg chamber in which BCs have migrated in advance of the follicular epithelium. The oocyte extends to meet the BCs while the BCs extend to meet the oocyte (arrowheads). These excessive interactions between the oocyte and follicle cells are never observed in wild-type egg chambers.

niscent of the cytoplasmic extensions observed between posterior germ cells and invasive follicle cells in egg chambers mutant for stronger dlg mutations (Fig. 4c,d).

kek-1 is expressed in BCs and in overproliferating and invasive follicle cell populations

Our analysis indicates that follicle cells at the poles of egg chambers throughout the growth phases, and BCs during the migratory phases, respond differentially to loss of dlg during oogenesis. This finding was unexpected because DIg protein is expressed at uniform levels in all follicle cells throughout oogenesis (Fig. 5). To determine if follicle cells sensitive to loss of DIg function might be genetically distinct, we screened enhancer trap lines. We found that the Drosophila kekkon-1 (kek-1) gene (M ussachio and Perrimon 1996) is specifically expressed in fields of follicle cells at the poles of the egg chamber, and in BCs before and during their migrati on to the oocyte (Fig. 9). This finding suggests that BCs and follicle cells at the poles of the egg chamber are functionally distinct, on the basis of sensitivity of these cells to loss of DIg function, and genetically distinct, on the basis of their expression of the kek-1 gene.

\section{Discussion}

DIg acts in a developmental pathway that prohibits the cell shape changes and morphogenetic transitions characteristic of BC migration

We show for the first time that DIg is required to prohibit cell invasion, in a pattern resembling border cell migration. BCs are distinguished from other follicle cells by expression of a C/EBP-like factor, encoded by the sl bo gene (M ontell et al. 1992). Following C/EBP expression, BC s become further distinguished by their adoption of an apolar morphology, their migration, and their interaction with germ cells in the posterior of the egg chamber (Fig. 1). We found that the DIg plays no role in BC specification, but rather is likely to function following C/EBP expression to regulate morphogenetic activities of both BCs and germ cells. In support of this hypothesis, we find that BCs migrate prematurely in animals mutant for weak DIg mutations (Fig. 8). Premature migration may result from both decreased DIg activity between BCs and adjacent follicle cells, as indicated by the expression of DIg at sites of contact between these cells (Fig. 5), and from the excessive interaction of the BCs with the germs cells, as indicated by the hypertrophic cell extensions between BCs and the oocyte (Fig. 8). We speculate that simultaneous reduction and loss of DIg activity in BCs and germ cells, respectively, allows BC migration in wild-type animals. BC differentiation, induced by slbo, may lead to reduction of DIg activity specifically in BCs (see bel ow), whereas unknown factors apparently lead to a loss of Dig protein in germ cells (Fig. 5).

That reduction of DIg in germ and follicle cells is essential for BC migration is indicated by the cellular invasion phenotype observed when DIg activity is lost or reduced in both germ cells and follicle cells, starting as early as sl of oogenesis. The cellular invasion phenotype is associated with several cellular events indistinguishable from those guiding BC movement. Both BC s and dlg invasive cells undergo an epithelial-mesenchyme-like transition, in which they move from adjacent epithelial cells as they shift their morphology from a polarized epithelial phenotype to an apolar morphology. dlg invasive follicle cells then move to the oocyte, along a path that is indistinguishable from the BC pathway. Invasive cells never turn from the central path, between lateral nurse cell membranes. The fidelity of this migration pattern is likely to result from specific interaction of invasive cells with posterior nurse cells, as indicated by the hyperplastic extension of a cytoplasmic process by a nurse cell adjacent to the oocyte (Fig. 4), resembling the contacts 


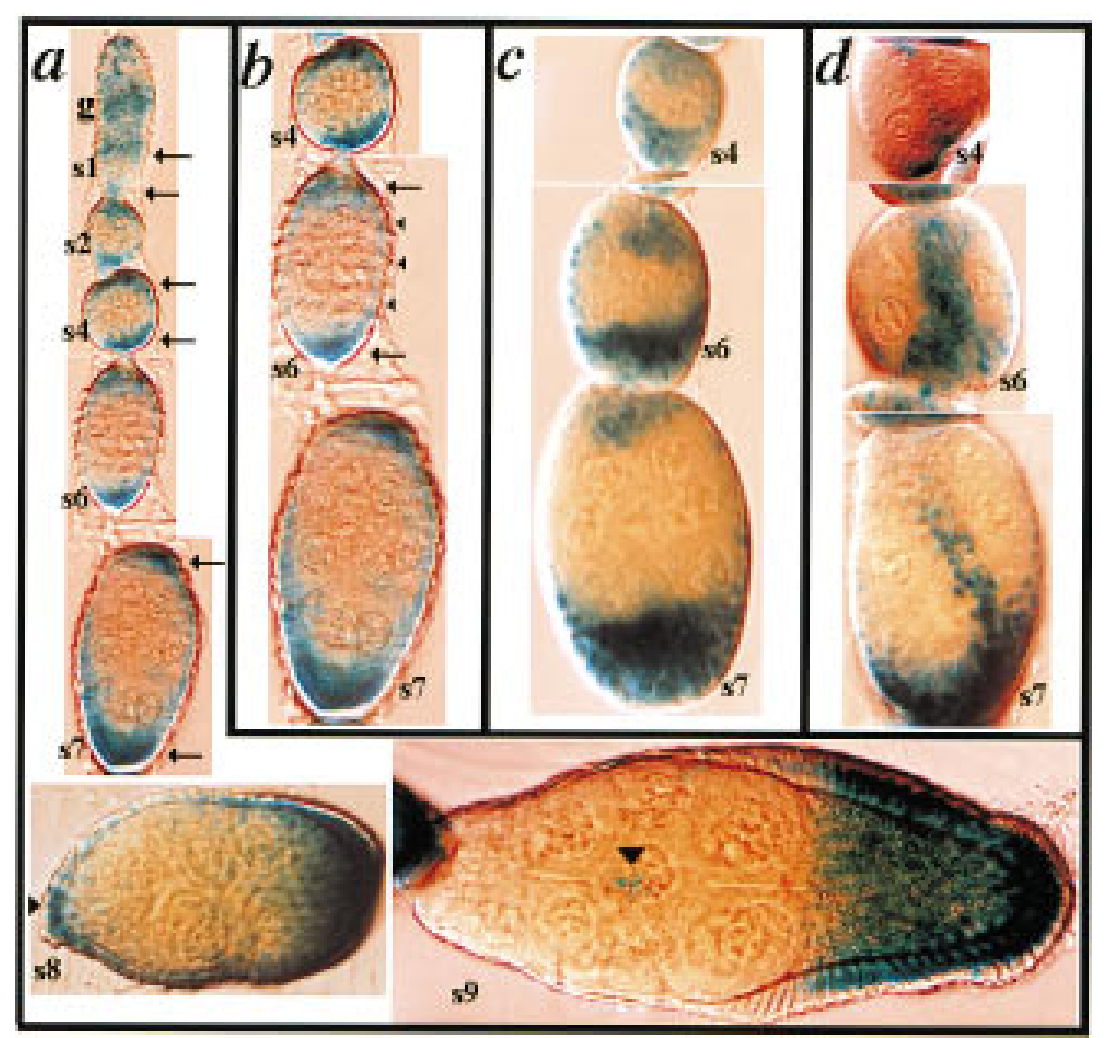

Figure 9. kek-1 is expressed in follicle cells sensitive to loss of dlg activity. Egg chambers harbor a single copy of a lacZ enhancer trap gene inserted in the $5^{\prime}$ noncoding region of kek-1 (M usacchio and Perrimon 1996). kek-1 expression is revealed by $X$-gal staining. (a) kek-1 is intensely expressed at the anterior and posterior poles of egg chambers throughout the time that follicle cells are proliferating, from s1 to s6, and continuing to s7 (arrows). At s8, kek-1 is expressed in the presumptive BCs at the anterior of the egg chamber (arrowhead). At s9, kek-1 is expressed in migrating $\mathrm{BC}$, and in follicular epithelial cells covering the oocyte, but not in nurse cell-associated epithelial cells. (b) Higher magnification of s4-s7 egg chambers shown in (a). In addition to polar staining, kek-1 is expressed at lower levels in lateral follicle cells (arrowheads). (c) Multiple layers of follicle cells at the anterior and posterior poles of $\mathrm{dlg}^{\text {sw }}$ egg chambers express kek-1. (d) Invasive follicle cells in $\mathrm{dlg}^{\text {hf } 321} / \mathrm{dlg}^{\mathrm{lv} 55}$ egg chambers express kek-1. established between BCs and an oocyte-adjacent nurse cell during normal oogenesis (Fig. 1). Loss or reduction of DIg appears to be required in both cell types to permit these interactions, because the invasive phenotype is always manifested when dlg activity is eliminated or reduced in both germ cells and follicle cells, but is typically not manifested when one tissue is mutant. This functional requirement is consistent with morphological evidence indicating that both germ and follicle cells play a role in guiding $B C$ movements.

Significantly, our findings imply that DIg does not simply inhibit individual cell behaviors during oogenesis, but rather may be a component in a devel opmental pathway essential for blocking cell proliferation and migration in a spatio-temporally defined pattern. dlg mutant egg chambers indicate that this pathway acts in germ and BCs and in fields of follicle cells at the anterior and posterior poles of egg chambers. Functionally, this is indicated by anterior and posterior follicle cell shape change, overaccumulation, and movement towards the oocyte (Figs. 2, 6-8; the movement of apolar posterior cells towards the oocyte is best documented in Fig. 2e), and genetically, this is indicated by kek-1 expression in BCs and follicle cells at the poles of the egg chamber. Strikingly, sl bo is also expressed at both the anterior and posterior poles of the egg chamber (Fig. 3), further suggesting a relationship between polar follicle cells.

$\mathrm{BC} s$ and dlg invasive cells are distinguished in that the latter are supplied from a field of overproliferating follicle cells that migrate as a continuous stream, whereas
$\mathrm{BCs}$, derived from a postmitotic epithelium, completely dissociate from the follicular epithelium. These distinctions result from the requirement of DIg in proliferation control (see below), and from the inability of dlg mutant cells to completely break from the follicular epithelium. These two processes appear to be intimately connected. In animals harboring dlg temperature-sensitive mutations, follicle cell overproliferation and cell invasion can be turned off simply by placing the animals at the permissive temperature (not shown). Under these circumstances, the epithelium heals, while invading cells continue to move towards the oocyte. Thus, the breaking of BCs from the follicular epithelium during wild-type oogenesis may involve a cycle of reduced DIg activity for the BCs to exit the epithelium, followed by a return to higher activity to reseal the follicular epithelium.

Whereas we have presented several lines of positive evidence implicating DIg in a pathway that inhibits the invasion characteristic of BCs, specificity implies that DIg should not be required in many other cellular interactions during oogenesis. Both anterior-posterior and dorsal-ventral specification of follicle cells, which establish the oocyte axes, are known to depend on intimate interactions between germ and follicle cells (GonzalezReyes et al. 1995; Roth et al. 1995), yet we do not observe any defects in these processes in dlg mutant egg chambers. Furthermore, we have not observed a role for DIg in the movement of the follicul ar epithelium al ong the outside of the egg chamber during s9. M any aberrant interactions between germ cells and follicle cells could be 
imagined, such as simultaneous invasion of follicle cells between the interface of each nurse cell, but such patterns are not observed. Finally, we suggest that the requirement for DIg in prohibiting follicle cell proliferation may not be distinct from dlg functions maintaining epithelial integrity, because the loss of epithelial characteristics of follicle cells during the early stages of oogenesis occurs in the environment of a mitotically active epithelium. Thus, the requirement of DIg for inhibiting follicle cell division may reflect an indirect consequence of the protein's requirement in maintaining appropriate contact between the epithelial cells, which may be indirectly tied to the regulation of follicle cell proliferation.

DIg PDZ domains appear to be required

for prohibiting follicle cell invasion

We found that the DIg SH3 and GuK domains do not appear to play a role in blocking cell invasion (Fig. 7), indicating that the PDZ domains inhibit germ cell-follicle cell mixing. We have not tested this hypothesis directly, because dlg mutations that specifically disrupt the PDZ domains do not exist. What role might the PDZ domains play in blocking cell mixing? PDZ domains bind to the cytoplasmic tail of transmembrane molecules and localize them to specific regions on the cell surface (for review, see Anderson 1996; Sheng 1996). During oogenesis, DIg is localized to sites of contact between germ cells and to sites of contact between follicle cells, but DIg is absent from sites of contact between germ cells and follicle cells (Fig. 5). This localization almost certainly ensures that presumptive Dlg-bound cell surface molecules retain tissue-specific segregation, and further suggests that DIg PDZ domains serve as components of a germ cell-foll icle cell tissue barrier (Fig. 10). Because the germ cell-follicle cell interactions that we observe in dlg mutant egg chambers specifically resemble BC interactions (see previous section), we suggest that DIg binds to a class of cell surface molecules that have the potential to mediate the cellular interactions characteristic of BC development, starting at s1 (Fig. 10). We propose that at the time of BC migration, via a regulated step that allows the redistribution of at least some of the cell surface molecules bound by DIg PDZ domains to the interface between germ cells and follicle cells, germ cells and BCs intermingle. Obvious candidates for this regulated step are loss or reduction of DIg in germ cells, and expression of slbo in BCs. Loss or reduction of DIg in germ cells might lead to the diffusion of previously bound molecules to cellular regions other than the interface with other germ cells. We can only speculate how slbo might elicit a freeing of DIg bound molecules, but precedent for regulated interactions involving MAGuKs is the interaction between the MAGuK PSD-95 and the inward rectifier $\mathrm{K}^{+}$channel Kir 2.3, which appears to be blocked by phosphorylation of a serine within the consensus PDZ-binding motif of Kir 2.3 by protein kinase A (Cohen et al. 1996). We suggest that in dlg mutant egg chambers, molecules bound by

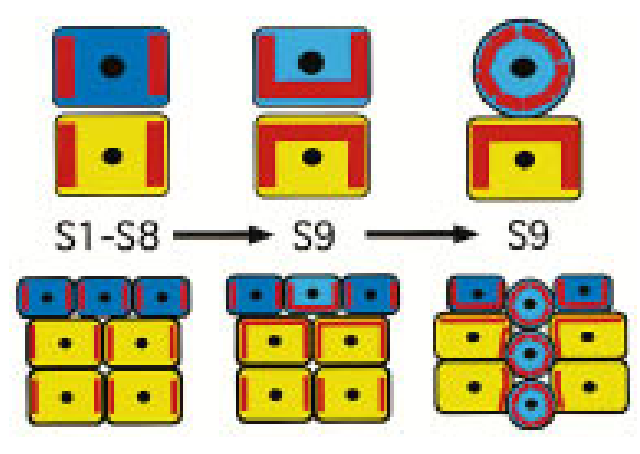

Figure 10. Model for Dlg in prohibiting cell invasion during oogenesis. Germ cells are yellow and follicle cells are blue. The red stripes within in each cell represent the distribution of putative membrane spanning proteins held in place by DIg PDZ domains (see text). During s1 to s8 of oogenesis, Dlg restricts these putative membrane spanning proteins to sites of contact between germ cells and to sites of contact between follicle cells, as indicated by the local ization of DIg protein during oogenesis. We propose that at s9, differentiation of BCs (light blue), leads to a redistribution of at least some DIg-bound molecules to sites of contact between germ and follicle cells, whereas loss or reduction of DIg in germ cells has a similar consequence. The interaction of membrane spanning molecules on the surface of germ and follicle cells then contributes to the driving force for BC invasion between germ cells. In dlg mutant egg chambers, the redistribution of PDZ-bound molecules happens in the absence of specific signals, leading to invasion as early as sl of oogenesis.

PDZ domains are freed prematurely, in the absence of specific signals, resulting in patterns of cell transformation and intercellular interaction resembling BC development. This model suggests that the BC migration pathway has been establi shed very early in oogenesis and provides an explanation for the variegated adhesion phenomena mediated by DIg, apparently promoting adhesion between follicle cells, while inhibiting contact and excessive interaction between germ cells and follicle cells.

DIg may help to pattern follicle cell divisions

Loss or reduction of DIg activity in the follicular epithelium causes follicle cells to overaccumulate at the anterior and posterior poles of the egg chamber (Figs. 2, 6, 7, and 9). We do not believe that this overaccumulation results from redistribution of follicle cells, because we did not find discontinuities in lateral regions of the epithelium (Figs. 2, 6, 7, and 9). Further, egg chambers appear to have a greater total number of follicle cells, and follicle cells incorporate BrdU higher levels in the regi on of the epithelium that contains overaccumulating cells (Fig. 7). The spatial specificity of inhibited follicle cell proliferation does not reflect an underlying pattern in follicle cell divisions, because follicle cells in the wild- 
Dlg inhibits cell invasion

type follicular epithelium appear to enter both $\mathrm{S}$ phase and $M$ phase at a uniform frequency relative to the anterior-posterior axis of the egg chamber (this study; Goode et al . 1996b). Rather, the differential requirement of Dlg would appear to reflect an underlying distinction between follicle cells at the poles of the egg camber. The fields of cells at the poles are distinguished by their expression of kek-1, and perhaps by greater intercellular tension caused by their position in a curved region of the epithelium. DIg may be a component of a signaling/adhesion pathway that helps to establish a spatially uniform rate of cell proliferation by differentially inhibiting cell proliferation at the egg chamber poles.

\section{A Drosophila model for cellular invasion}

How do dlg invasive phenotypes compare with normal and pathological cell invasions in other organisms? The cell invasions that we have analyzed have no relevance to those in which cells traverse extracellular matrix, because basement membrane does not exist between either nurse cells or between the follicular epithelium and nurse cells. Rather, our data may be useful for understanding how cells move between heterologous cells during devel opmental processes such as migration of gonocytes, adult processes such as movement of leukocytes to specific destinations throughout the organism, and during pathological states such as cancer. Whereas most malignant cancer cells traverse an extracellular matrix before reaching their destination, some cancerous cells, particularly in the central nervous system, apparently metastasize strictly through cellular environments, such as axon fascicles (Mareel et al. 1991).

Regardless of interactions with matrices, at a cellular level, normal and pathological cell invasions involve common transitions, including loss of homotypic cell adhesion, epithelial-mesenchymal conversion, gain of heterotypic cell adhesion, and cell migration. In our studies, we have shown that DIg plays a role in mediating all of these cell behaviors. These cell activities provide a common link between normal and pathological cell invasions, and have led to the suggestion that developmental genes that block invasion are switched off in malignant cells, whereas genes that promote invasion become activated on devel opmental maturation ( $M$ areel et al. 1991). Although our developmental studies do not make a direct connection to adult malignancy, human DIg has been implicated in the development of the tumorous state via its association with APC, the most commonly mutated gene in human colon cancer (Matsumine et al. 1996). Further, we have shown that dlg invasive cells resemble malignant cells in several regards, including loss of homotypic adhesion, loss of proliferation control, and cell polarity, and enhanced heterotypic interactions and migratory ability. The DIg invasive phenotype may serve as an excellent genetic and cell biological model for analyzing intercellular interactions underlying cell shape and cell mixing phenomena in a variety of normal and pathological cell invasions.

\section{Materials and methods}

DIg mutations

dlg mutations used in this study have been described previously (Table 1; Perrimon 1988; Woods and Bryant 1989, 1991). All flies were reared on standard media at $25^{\circ} \mathrm{C}$ or were reared at $18^{\circ} \mathrm{C}$, and then shifted to $25^{\circ} \mathrm{C}$, as described in the text.

\section{Histochemistry}

Fluorescein-phalloidin staining was performed as described (Goode et. 1996a). Immunohistochemistry, by use of FITC-conjugated secondary antibodies, was performed as described previously (Goode et al. 1996b). The following sera were used in this study: DIg (Woods and Bryant 1991), Arm (Peifer et al. 1993), and FasllI (Patel et al. 1987). All fluorescein-labeled tissues were analyzed with a Bio-Rad laser scanning confocal microscope, attached to a Zeiss Axiophot microscope.

BrdU labeling and analysis of follicle cell divison patterns

BrdU was incorporated into ovarian tissue in vivo. Females that had been reared at $18^{\circ} \mathrm{C}$ were placed with an equal number of males for a few days and starved for $2 \mathrm{hr}$ at $18^{\circ} \mathrm{C}$ (to induce hunger). The flies were then shifted to $25^{\circ} \mathrm{C}$ onto a yeast paste mixture consisting of 0.5 grams of dry yeast: $1.5 \mathrm{ml}$ of BrdUlabeling solution (Amersham). After feeding for $12 \mathrm{hr}$, females were sacrificed and their ovarian tissue was fixed in Carnoy's fixative, washed in PBT, hydrolyzed in $2 \mathrm{~N} \mathrm{HCl}$ for $1 \mathrm{hr}$, and rinsed twice in PBT. Ovarian tissue was then treated with 50 $\mu \mathrm{g} / \mathrm{ml}$ of RN ase in PBS for $2 \mathrm{hr}$. Following RN ase, the tissue was stained with antibodies to BrdU (Amersham), by use of a FITCconjugated secondary antibody, as described above. The tissue was then stained for $20 \mathrm{~min}$ with propidium iodide and rinsed with PBS. The relative position of follicle cells that had incorporated BrdU was established with a laser scanning confocal microscopy, as described previously (Goode et al. 1996b).

Clonal analysis

To analyze the phenotypic consequence of introducing dlg mutations in germ cells, follicle cells, or both tissues simultaneously, FRT-mediated recombination was induced with a heat shock FLPase (hsFLPase) chromosome (Golic and Lindquist 1989). Clones were marked by loss of a constitutively expressed lacZ gene driven by a tubulin promoter, TUBlacZ [(Fig. 6; originally X37 IacZ (D. Harrison, pers. comm.)]. A nuclear localization signal is fused to the $\beta$-gal protein produced by the $X 37$ lac $Z$ gene, thus targeting expression to both germ and follicle cell nuclei. FRT ${ }^{101}$ was used in all experiments. The progeny of dlg $\mathrm{FRT}^{101} / \mathrm{FM} 7$, B animals crossed to TUBlacZ FRT ${ }^{101} / \mathrm{Y}$; hsFLPase/ + animals were heat shocked for $2 \mathrm{hr}$ on consecutive days during early pupal development. Adult $\mathrm{B}^{+}$females were allowed to lay eggs for several days before dissecting ovaries. Ovaries were stained for lacZ expression by standard methods.

Western analysis

Western analysis was performed as described (Woods et al. 1996).

\section{Acknowledgments}

Dan Woods provided DIg sera and stimulating and helpful discussion throughout this study. We thank Kevin Fitzgerald, Denise Montell, and Dan Woods for hel pful comments on the 
manuscript. We thank Beth $\mathrm{N}$ oll and Betsy Wilder for technical advice concerning the BrdU labeling protocol and Allan Spradling for helpful discussion concerning follicle cell counts. Doug Harrison kindly provided the X37 tubulin lacZ marker. Arm sera were a gift of $M$ ark Peiffer, and FasllI sera were a gift of N ipam Patel. S.G. was supported by a $\mathrm{N}$ ational Institutes of Health postdoctoral fellowship (GM 17511-02) and is currently a Leukemia Society of America Special Fellow (3232-98). N .P. is an investigator of the Howard Hughes Medical Institute.

The publication costs of this article were defrayed in part by payment of page charges. This article must therefore be hereby marked "advertisement" in accordance with 18 USC section 1734 solely to indicate this fact.

\section{References}

Anderson, J.A. 1996. Cell signaling: MAGUK magic. Curr. Biol. 4: 382-384.

Birchmeier, W. and J. Behrens. 1994. Cadherin expression in carcinomas: Role in the formation of cell junctions and the prevention of invasiveness. Biochim. Biophys. Acta 1198: 11-26.

Cohen, N.A., J.E. Brenman, S.H. Snyder, and D.S. Bredt. 1996. Binding of the inward rectifier $\mathrm{K}^{+}$channel Kir 2.3 to PSD-95 is regulated by protein kinase $A$ phosphorylation. Neuron 17: 759-767.

Gateff, E. 1978. Malignant neoplasms of genetic origin in Drosophila melanogaster. Science 200: 1448-1459.

Golic, K.G. and S. Lindquist. 1989. The FLP recombinase of yeast catalyzes site-specific recombination in the Drosophila genome. Cell 59: 499-509.

Gonzalez-Reyes, A., H. Elliott, and R.D. St. Johnston. 1995. PoIarization of both major body axes in Drosophila by gurkentorpedo signalling. Nature 375: 654-658.

Goode, S., D. Wright, and A.P. M ahowald. 1992. The neurogenic locus brainiac cooperates with the Drosophila EGF receptor to establish the ovarian follicle and to determine its dorsalventral polarity. Development 116: 177-192.

Goode, S., M. Morgan, Y.-P. Liang, and A.P. M ahowald. 1996a. brainiac encodes a novel, putative secreted protein that $\mathrm{co}$ operates with grk TGF $\alpha$ to produce the follicular epithel ium. Dev. Biol. 178: 35-50.

Goode, S., M. Melnick, T.-B. Chou, and N. Perrimon. 1996b. The neurogenic genes egghead and brainiac define a novel signaling pathway essential for epithelial morphogenesis during Drosophila oogenesis. Development 122: 3863-3879.

Gumbiner, B.M. 1996. Cell adhesion: The molecular basis of tissue architecture and morphogenesis. Cell 84: 345-357.

Hynes, R.O. and A.D. Lander. 1992. Contact and adhesive specificities in the associations, migrations, and targeting of cells and axons. Cell 68: 303-322.

Lahey, T., M. Gorczyca, X.X. Jia, and V. Budnik. 1994. The Drosophila tumor suppressor gene dl $g$ is required for normal synaptic bouton structure. Neuron 13: 823-835.

Mahowald, A.P. 1972. Ultrastructural observations on oogenesis in Drosophila. J. Morphol. 137: 28-48.

Mareel, M.M., P. De Baetselier, and F.M. Van Roy. 1991. Mechanisms of invasion and metastasis. CRC Press, Boca Raton, FL.

Matsumine, A., O. Akiko, S. Tadao, N. Okumura, K. Satoh, B. Gyeong-Hun, T. Kawahara, S. Kobayashi, M. Okada, K. Toyoshima, and T. Akiyama. 1996. Binding of APC to the human homolog of the Drosophila discs large tumor suppressor protein. Science 272: 1020-1023.

Mayer, B.J. and M.J. Eck. 1995. SH3 domains. Minding your p's and q's. Curr. Biol. 5: 364-367.

Montell, D.J. 1994. Moving right along: Regulation of cell migration during Drosophila development. Trends Genet. 10: 59-62.

Montell, D.J., P. Rorth, and A.C. Spradling. 1992. slow border cells, a locus required for a developmentally regulated cell migration during oogenesis, encodes Drosophila C/EBP. Cell 71: 51-62.

Murphy, A.M., T. Lee, C.M. Andrews, B.Z. Shilo, and D.J. Montell. 1995. The breathless FGF receptor homolog, a downstream target of Drosophila C/EBP in the developmental control of cell migration. Development 121: 2255-2263.

M usacchio, M. and N. Perrimon. 1996. The Drosophila kekkon genes: Novel members of both the leucinerich repeat and immunoglobulin superfamilies expressed in the CNS. Dev. Biol. 178: 63-76.

Patel, N.H., P.M. Snow, and C.S. Goodman. 1987. Characterization and cloning of fasciclin III: A glycoprotein expressed on a subset of neurons and axon pathways in Drosophila. Cell 48: 975-988.

Peifer, M., S. Orsulic, D. Sweeton, and E. Wieschaus. 1993. A role for the Drosophila segment polarity gene armadillo in cell adhesion and cytoskeletal integrity during oogenesis. Development 118: 1191-1207.

Perrimon, N. 1988. The maternal effect of I(1)discs-large: A recessive oncogene of Drosophila melanogaster. Dev. Biol. 127: 392-407.

Roth, S., F.S. N euman-Sil berberg, G. Barcel o, and T. Schupbach. 1995. cornichon and the EGF receptor signaling process are necessary for both anterior-posterior and dorsal-ventral pattern formation in Drosophila. Cell 81: 967-978.

Sheng, M. 1996. PDZs and receptor/channel clustering: Rounding up the latest suspects. Neuron 17: 575-578.

Simske, J.S., S.M. Kaech, S.A. Harp, and S.K. Kim. 1996. LET-23 receptor local ization by the cell junction protein lin-7 during C. el egans vulval induction. Cell 85: 195-204.

Songyang, Z., A.S. Fanning, C. Fu, J. Xu, S.M. Marfatea, A.H. Chishti, A. Crompton, A.C. Chan, J.M. Anderson, and L.C. Cantley. 1997. Recognition of unique carboxyl-terminal motifs by distinct PDZ domains. Science 275: 73-77.

Spradling, A.C. 1993. Developmental genetics of oogenesis. In The development of Drosophila melanogaster (ed. M. Bate and M. Arias), pp. 1-70. Cold Spring Harbor Laboratory Press, Cold Spring Harbor, NY.

Takeichi, M. 1988. The cadherins: Cell-cell adhesion molecules controlling animal morphogenesis. Development 102: 639655.

Woods, D.F. and P.J. Bryant. 1989. Molecular cloning of the lethal (1) discs large-1 oncogene of Drosophila. Dev. Biol. 134: 222-235.

- - 1 1991. The discs-large tumor suppressor gene of Drosophila encodes a guanylate kinase homolog local ized at septate junctions. Cell 66: 451-464.

-_- 1993. ZO-1, DIgA and PSD-95/SA P90: Homologous proteins in tight, septate, and synaptic cell junctions. Mech. Dev. 44: 85-89.

Woods, D.F., C. Hough, D. Peel, G. Callaini, and P.J. Bryant. 1996. DIg protein is required for junction structure, cell polarity, and proliferation control in Drosophila epithelia. J. Cell Biol. 134: 1469-1482. 


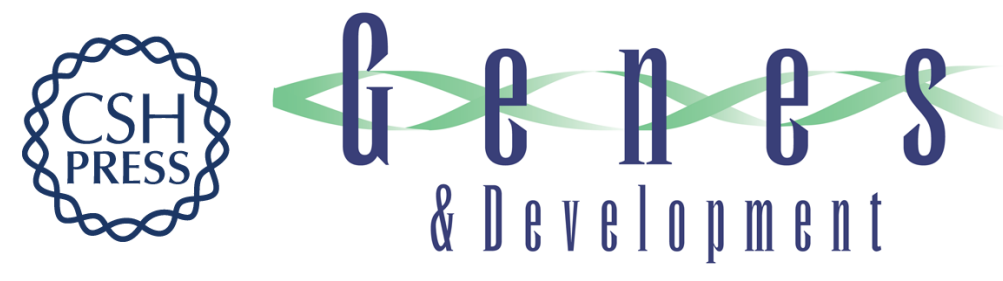

\section{Inhibition of patterned cell shape change and cell invasion by Discs large during Drosophila oogenesis}

Scott Goode and Norbert Perrimon

Genes Dev. 1997, 11:

Access the most recent version at doi:10.1101/gad.11.19.2532

References This article cites 30 articles, 9 of which can be accessed free at:

http://genesdev.cshlp.org/content/11/19/2532.full.html\#ref-list-1

License

Email Alerting

Receive free email alerts when new articles cite this article - sign up in the box at the top

Service right corner of the article or click here.

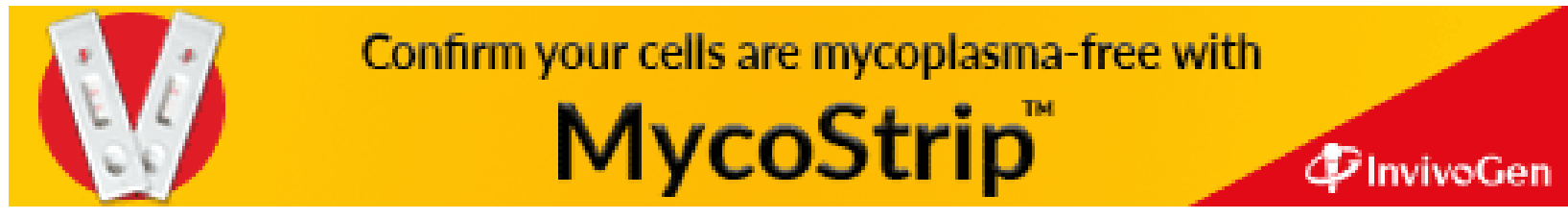

